\title{
Outcomes Following Re-irradiation for Symptomatic Brain Metastasis
}

\section{Ji Hoon Choi*}

Department of Radiation Oncology, Kosin University Gospel Hospital, Kosin University College of Medicine, Busan, Korea

\begin{abstract}
We retrospectively analyzed outcomes of patients who underwent reirradiation for brain metastasis. We analyzed the records of 23 patients with brain metastasis who were initially treated with palliative brain radiotherapy (RT) and were retreated with a second course of brain RT between June 2008 and December 2012. WBRT, 3DCRT, and SRS were used for brain metastasis. The median dose of the first course of WBRT was 30 Gy (range, 23.4-30 $\mathrm{Gy}$ ). The dose of the first course of 3DCRT for the lesion was $30 \mathrm{~Gy}$ in $3 \mathrm{~Gy}$ per fraction. The median dose of the first course of SRS was 16 Gy in one fraction (range, 12-24 Gy). The median dose of the second course of WBRT was $27.5 \mathrm{~Gy}$ (range, 12-30 Gy). The median dose of the second course of 3DCRT for the lesion was $30 \mathrm{~Gy}$ (range, 25-30 Gy). The dose of the second course of SRS was $16 \mathrm{~Gy}$ in one fraction. The second course of WBRT was administered on the basis of radiographically proven disease progression with clinical symptoms. With a median follow-up of 25 months, the overall symptom resolution rate was $47.8 \%$. The rate of palliative efficacy was $82.6 \%$ including patients with stable disease. The median survival time after initiation of reirradiation was 3.2 months. The median Karnofsky performance status (KPS) value prior to reirradiation was 30 , and the median KPS value following reirradiation was 60 . Reirradiation of brain metastasis is feasible and effective in select patients with performance status of KPS $\geq 60$ (Eastern Cooperative Oncology Group (ECOG) 0-2) prior to reirradiation.
\end{abstract}

Keywords: Brain metastasis; Reirradiation; Performance status; KPS

\section{Abbreviations}

RT: Radiotherapy; WBRT: Whole Brain Radiotherapy; KPS: Karnofsky Performance Status; 3DCRT: three-Dimensional Conformal Radiotherapy; SRS: Stereotactic Radiosurgery

\section{Introduction}

Metastatic brain tumors are 10 times more common than primary brain tumors, occurring in $24-45 \%$ of all cancer patients [1]. As the life expectancy of cancer patients has increased with improvements in chemotherapy, surgery, and radiotherapy treatment for lung and breast cancer, the incidence of brain metastasis has also increased [2,3]. Lung and breast cancer often spread to the brain, more often resulting in a terminal outcome. Such patients should be considered for appropriate treatment with radiation therapy or surgery [4].

As the life expectancy of cancer patients has increased with the development of chemotherapy, surgery, and radiotherapy, the possibility of treatment for metastatic brain tumor has increased as well. Patients with neurologic symptoms including headache, visual disturbance, and ataxia can be analyzed with brain CT or brain MRI. Proper radiotherapy of brain metastasis can offer benefits to survival in some patients, as well as palliation of symptoms [5].

Patients with brain metastasis undergo palliative radiotherapy. When these patients suffer disease progression with neurologic symptoms, a second course of irradiation should be considered [68]. Reirradiation of the brain lesion remains controversial because of treatment-related side effects and questions regarding palliative efficacy $[9,10]$. We analyzed the outcomes of brain reirradiation incranial metastasis patients at our institution in order to assess the factors for patient selection.

\section{Methods and Materials}

\section{Patients}

This retrospective study included 23 patients with brain metastasis who underwent reirradiation between June 2008 and December
2012 at the Kosin University Gospel Hospital. Inclusion criteria were patients with pathologically proven breast cancer or lung cancer who were initially treated for measurable metastatic brain lesion with neurologic symptoms. The most common primary disease site was lung (11 patients with non-small-cell lung cancer, four with small-cell lung cancer), followed by breast (eight patients). No patients received surgical resection for brain lesions. Survival time was defined as the time from the start of re-irradiation to the date of death using the Kaplan-Meier method. Statistical analysis was carried out using the log-rank test to test whether there was a difference between the survival times of different groups of patients and for a variety of treatment factors. Extracranial disease status was determined by official radiologic evaluation. Patient characteristics are listed in Table 1.

\section{Treatment}

Treatment details are listed in Table 2. The chosen three radiotherapy techniques for brain metastasis were whole brain radiotherapy (WBRT), three-dimensional conformal radiotherapy (3DCRT) of partial brain radiotherapy, and stereotactic radiosurgery (SRS). The median dose of the first course of WBRT was $30 \mathrm{~Gy}$ in $3 \mathrm{~Gy}$ per fraction (range, 23.4-30 Gy). The dose of the first course of partial brain RT was $30 \mathrm{~Gy}$ in $3 \mathrm{~Gy}$ per fraction. The median dose of the first course of SRS was $16 \mathrm{~Gy}$ in one fraction (range, 12-24 Gy). The median dose of the second course of WBRT was 27.5 (range, 12-30 Gy). The median dose of the second course of 3DCRT was 30 Gy (range, 25-30

*Corresponding author: Ji Hoon Choi, Department of Radiation Oncology, Kosin University Gospel Hospital, Kosin University College of Medicine, 262, Gamcheonro, Seo-gu, Busan, 602-702, Korea, Tel: +82-51-990-6399; Fax: +82-51-793-0660; E-mail: 1stdr@naver.com

Received September 25, 2015; Accepted October 17, 2015; Published October 26, 2015

Citation: Choi JH (2015) Outcomes Following Re-irradiation for Symptomatic Brain Metastasis. J Cancer Sci Ther 7: 308-311. doi:10.4172/1948-5956.1000368

Copyright: (C) 2015 Choi JH. This is an open-access article distributed under the terms of the Creative Commons Attribution License, which permits unrestricted use, distribution, and reproduction in any medium, provided the original author and source are credited. 
Gy). The dose of the second course of SRS was 16 Gy in one fraction (Table 2).

\section{Palliative treatment efficacy}

The analyzed response criteria were relief of symptoms, stable status, or aggravation of symptoms. Using medical records, Karnofsky performance status (KPS) prior to and after re-irradiation were obtained.

\section{Results}

The most common RT technique was WBRT as the initial course followed by 3DCRT for reirradiation in eight (34.8\%) of 23 patients. Among patients who underwent WBRT, three received WBRT for reirradiation. The mean time interval between the two courses of brain RT was 13.6 months (median, 11.1; range, 1-38).

Palliative efficacy defined as relief of symptoms is listed in Table 3. Of 23 total patients, $11(47.8 \%)$ experienced better than partial resolution of symptoms, while eight did not experience any symptom improvement. Four (17.3\%) patients demonstrated symptom aggravation.

Performance in the first and second courses of RT was compared. Prior to repeat brain RT, the mean KPS was 42.2 (median, 30), while that following irradiation was 52.7 (median, 60). At the three-month follow-up, eight patients were deceased following disease progression, and the mean KPS before re-irradiation was 32.5 (median, 30). Two patients died of progressive primary disease during the course of reirradiation.

The median overall survival after reirradiation of brain metastases

\begin{tabular}{|c|c|}
\hline Parameter & No. of patients $(n=23)$ \\
\hline \multicolumn{2}{|l|}{ Gender } \\
\hline Male & 7 \\
\hline Female & 16 \\
\hline \multicolumn{2}{|l|}{ Age (years) } \\
\hline Median (range) & $54(43-67)$ \\
\hline \multicolumn{2}{|l|}{ Primary tumor } \\
\hline Breast & 8 \\
\hline \multicolumn{2}{|l|}{ Lung } \\
\hline NSCLC & 11 \\
\hline SCLC & 4 \\
\hline \multicolumn{2}{|c|}{ KPS before re-irradiation } \\
\hline $100 \sim 70$ & 4 \\
\hline $60 \sim 40$ & 7 \\
\hline$<30$ & 12 \\
\hline \multicolumn{2}{|c|}{ RPA classification } \\
\hline I & 3 \\
\hline II & 1 \\
\hline III & 19 \\
\hline
\end{tabular}

Table 1: Clinical patient characteristics.

\begin{tabular}{|c|c|c|}
\hline Firstcourse of RT & Reirradiation & No. of patients \\
\hline WBRT & 3D CRT & 8 \\
\hline WBRT & WBRT & 3 \\
\hline WBRT & SRS & 2 \\
\hline 3D CRT & 3D CRT & 2 \\
\hline 3D CRT & WBRT & 2 \\
\hline SRS & WBRT & 4 \\
\hline SRS & 3D CRT & 2 \\
\hline
\end{tabular}

Table 2: Radiation therapy details and number of patients per therapy.

\begin{tabular}{|c|c|}
\hline Response & No. of patients \\
\hline Relief of symptoms & $11(47.8 \%)$ \\
\hline Stable (maintaining) & $8(34.8 \%)$ \\
\hline Aggravation of symptoms & $4(17.4 \%)$ \\
\hline
\end{tabular}

Table 3: Palliative efficacy.

was 3.2 months (Figure 1). The median overall survival for RPA class I patients ( 3 of 23) was 16 months, compared with 25 months for RPA class II ( 1 of 23) and 3 months for RPA class III (19 of 23). There was a statistically significant correlation between survival and KPS value (Figure 2). The median overall survival for patients with favorable performance status (KPS $\geq 60$ ) was 16 months, compared with three months for patients with KPS less than $60(\mathrm{p}=0.015)$.

Acute and chronic toxicity were assessed according to the Common Toxicity Criteria for Adverse Events (CTCAE, version 4.0). No patients experienced radiation necrosis after the second course of brain RT. One (4.3\%) patient experienced a treatment-related Grade 3 headache.

\section{Discussion}

Secondary radiation therapy for brain metastasis remains a controversial intervention with potential challenges. Repeat irradiation concerns include toxicity and limited symptom palliation efficacy [11]. There are different reirradiation options depending on the initial brain irradiation technique. Several publications have evaluated the outcomes of repeat irradiation, and most studies have concluded that repeat irradiation is an effective intervention with tolerable side effects [12-18]. Son et al. [12] examined outcomes in patients at Massachusetts General Hospital who underwent WBRT as a reirradiation treatment option. The median dose of the first course of WBRT was $35 \mathrm{~Gy}$ (range, 28-40 Gy), with a fraction size of 2 to $3 \mathrm{~Gy}$ (median, $2.5 \mathrm{~Gy}$ ). The median dose at reirradiation was $21.6 \mathrm{~Gy}$ (range, $14-30 \mathrm{~Gy}$ ), with a fraction size of 1.5 to $2 \mathrm{~Gy}$ (median, $1.8 \mathrm{~Gy}$ ). The median overall survival after the second course of WBRT was 5.2 months. The median overall survival of patients with stable extracranial disease at the time of reirradiation was 19.8 months, compared with a median overall survival of 2.5 months for patients with progressive extracranial disease $(p=0.05)$. Sadikov et al. [19] reported that the time interval between two radiation courses ( $\leq$ 6 months vs. $6-11.9$ months vs. $\geq 12$ months) did not show significant differences in survival.

The protocol of our institution is to wait at least six months between brain reirradiation courses, except in rare patients who have worsening symptomatic brain metastasis after the initial RT course. This practice is premised on the concern of increased risk of neurological toxicity caused by the short time interval between consecutive radiation treatments. In our study, one (4.3\%) patient had a treatment-related grade 3 adverse event. The patient underwent two courses of 3D conformal radiotherapy for partial brain metastasis. The dose of the first course of RT was 30 Gy with a fraction size of $3 \mathrm{~Gy}$, followed by a reirradiation course of $25 \mathrm{~Gy}$ with a fraction size of $2.5 \mathrm{~Gy}$. The patient developed grade 3 headache associated with treatment. The cumulative biological equivalent doses (BEDs) for the patient were $105.8(\alpha / \beta=3$ Gy) and $70.2 \mathrm{~Gy}(\alpha / \beta=10 \mathrm{~Gy})$. The time between the two courses was four months.

Whole brain radiation therapy for the purpose of palliation remains controversial because of safety concerns. Emami et al. [20] reported normal organ tissue tolerance doses in various organs and tissues. These data were applied to a partial dose-volume model, and the TD $5 / 5$ (total radiation dose that can induce $5 \%$ of complications within five years) of the whole brain, $2 / 3$ of the brain, and $1 / 3$ of the brain were 


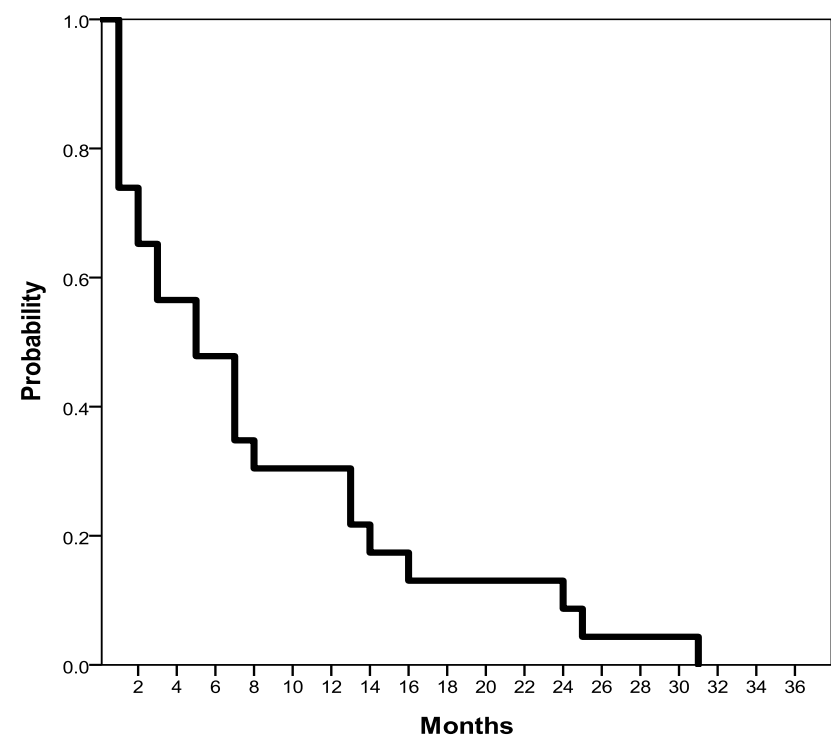

Figure 1: Overall survival after re-irradiation. The median overall survival was 3.2 months.

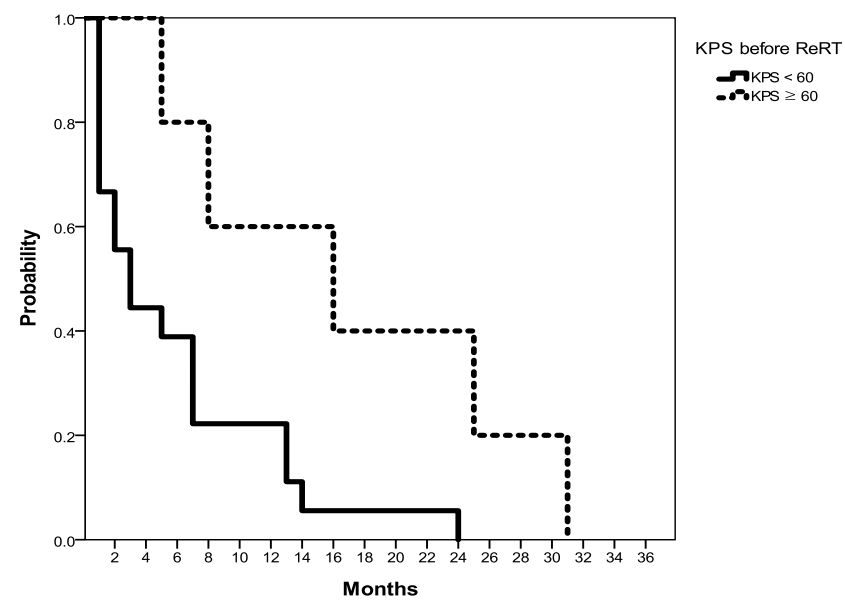

Figure 2: Overall survival following reirradiation according to KPS. The median overall survival for patients with KPS $\geq 60$ was 16 months and was three months for patients with KPS $<60$.

reported as 4,500 cGy, 5,000 cGy, and 6,000 cGy, respectively. Recently, the Quantitative Analysis of Normal Tissue Effects in the Clinic review [21] refined normal tissue dose volume tolerance guidelines for the brain in the context of the modern era of radiotherapy. In our study, three patients who underwent repeated WBRT did not tend to have worse survival or more treatment-related side effects than patients undergoing other treatment options. Repeat irradiation patients had more significant disease burden prior to re-irradiation. The toxicity results might be attributable to the fact that patients had worse disease and might have died before the development of late toxicity.

However, Son et al. [12] reported 17 patients who received a repeat course of WBRT and experienced no grade 3 toxicity effects. Mayer et al. [11] reported that radiation-induced normal brain tissue necrosis occurred at NTD cumulative $>100 \mathrm{~Gy}$. There was no association between irradiation course time interval and incidence of radionecrosis.

Prior studies have analyzed correlations between brain re- irradiation and historically favorable prognostic factors, including performance status (KPS >70), absence of extracranial disease, and age. Sadikov et al. [19] found that Eastern Cooperative Oncology Group (ECOG) performance status and Radiation Therapy Oncology Group (RTOG) neurologic function class were also significant prognostic factors. ECOG performance status before reirradiation was a strong prognostic factor of survival in patients with brain metastasis. Patients with ECOG performance status 0-1 showed better survival outcome (median 6.4 months) than patients with ECOG performance status 2-3 (2.0 months) ( $\mathrm{p} \leq 0.0001)$. Wong et al. [5] analyzed 86 patients, $47 \%$ of whom were ECOG 2 and $44 \%$ of whom were ECOG 3. Among all patients, performance status improved after re-irradiation in 30 patients (35\%) and remained unchanged in 42 patients $(49 \%)$. Various potential prognostic factors were evaluated for association with survival, two of which were statistically correlated with survival: absence of extracranial metastasis and solitary brain lesion. No association between performance status and survival was reported.

We retrospectively analyzed KPS prior to and after re-irradiation using patient medical records. In our study, the mean KPS of patients with brain metastasis was 42.2 (median, 30) prior to repeat brain RT, while that after re-irradiation was 52.7 (median, 60). Table 3 lists the assessment of patient responses following re-irradiation. Brain reirradiation was beneficial for symptom palliation. More than partial symptom relief, including complete relief, was observed in 11 (47.8) of 23 patients. Eight of the $23(34.7 \%)$ patients demonstrated stable symptoms, while four patients $(17.4 \%)$ experienced aggravation of symptoms. These aggravated disease patients were RPA class III and had primary disease progression status. The time interval between the two courses of irradiation was not a factor of palliative efficacy. We analyzed the association of segmented KPS and patient survival. There was a statistically significant correlation between survival and a KPS value of 60 (Figure 2). The median overall survival for patients with good performance status (KPS $\geq 60$ : ECOG 0-2) was 16 months, compared with three months for patients with KPS less than $60(\mathrm{p}=$ 0.015). In comparison with previous studies, the patient population in our study demonstrated a somewhat less favorable performance status, with a median initial KPS of 30, and included a majority of patients with lung cancer rather than breast cancer. We observed a trend for better survival in patients with primary breast cancerand with stable extracranial disease.

\section{Conclusion}

Within the limitations of a retrospective review, we found clinical benefit for re-irradiation in $82.6 \%$ of patients, including symptom maintenance, and a median survival of 3.2 months in patients with progression or relapse after an initial course of radiation therapy for brain metastasis.

This study demonstrates that brain re-irradiation is likely to be useful in patients with good performance status, with KPS $\geq 60$ (ECOG 0 -2) prior to re-irradiation, and with stable extracranial disease. In our study, even patients with poor prognostic factors experienced palliation efficacy with re-irradiation therapy. In conclusion, brain re-irradiation can offer symptom palliation efficacy with tolerable toxicity.

\section{References}

1. Nussbaum ES, Djalilian HR, Cho KH, Hall WA (1996) Brain metastases. Histology, multiplicity, surgery, and survival. Cancer 78: 1781-1788.

2. Barani IJ, Larson DA, Berger MS (2013) Future directions in treatment of brain metastases. Surg Neurol Int 4: S220-230. 
Citation: Choi JH (2015) Outcomes Following Re-irradiation for Symptomatic Brain Metastasis. J Cancer Sci Ther 7: 308-311. doi:10.4172/19485956.1000368

3. Gavrilovic IT, Posner JB (2005) Brain metastases: epidemiology and pathophysiology. J Neurooncol 75: 5-14.

4. Nieder C, Spanne O, Mehta MP, Grosu AL, Geinitz H (2011) Presentation, patterns of care, and survival in patients with brain metastases: what has changed in the last 20 years? Cancer 117: 2505-2512.

5. Wong J, Hird A, Zhang L, Tsao M, Sinclair E, et al. (2009) Symptoms and quality of life in cancer patients with brain metastases following palliative radiotherapy. Int J Radiat Oncol Biol Phys 75: 1125-1131.

6. Minniti G, Scaringi C, Lanzetta G, Bozzao A, Romano A, et al. (2014) Whole brain reirradiation and concurrent temozolomide in patients with brain metastases. J Neurooncol 118: 329-334.

7. Ozgen Z, Atasoy BM, Kefeli AU, Seker A, Dane F, et al. (2013) The benefit of whole brain reirradiation in patients with multiple brain metastases. Radiat Oncol 8: 186.

8. Wong WW, Schild SE, Sawyer TE, Shaw EG (1996) Analysis of outcome in patients reirradiated for brain metastases. Int $\mathrm{J}$ Radiat Oncol Biol Phys 34: 585-590.

9. Lucas JT, Colmer HG, White L, Fitzgerald N, Isom S, et al. (2015) Competing Risk Analysis of Neurologic versus Nonneurologic Death in Patients Undergoing Radiosurgical Salvage After Whole-Brain Radiation Therapy Failure: Who Actually Dies of Their Brain Metastases? Int J Radiat Oncol Biol Phys 92: 1008-1015.

10. Yuan H, Gaber MW, Boyd K, Wilson CM, Kiani MF, et al. (2006) Effects of fractionated radiation on the brain vasculature in a murine model: blood-brain barrier permeability, astrocyte proliferation, and ultrastructural changes. Int J Radiat Oncol Biol Phys 66: 860-866.

11. Mayer R, Sminia P (2008) Reirradiation tolerance of the human brain. Int $J$ Radiat Oncol Biol Phys 70: 1350-1360.

12. Son CH, Jimenez R, Niemierko A, Loeffler JS, Oh KS, et al. (2012) Outcomes after whole brain reirradiation in patients with brain metastases. Int $\mathrm{J}$ Radiat Oncol Biol Phys 82: e167-172.

13. Adkison JB, Tomé W, Seo S, Richards GM, Robins HI, et al. (2011) Reirradiation of large-volume recurrent glioma with pulsed reduced-dose-rate radiotherapy. Int J Radiat Oncol Biol Phys 79: 835-841.

14. Maranzano E, Trippa F, Casale M, Costantini S, Anselmo P, et al. (2012) Reirradiation of brain metastases with radiosurgery. Radiother Oncol 102 192-197.

15. Olson AC, Wegner RE, Rwigema JC, Heron DE, Burton SA, et al. (2012) Clinica outcomes of reirradiation of brain metastases from small cell lung cancer with Cyberknife stereotactic radiosurgery. J Cancer Res Ther 8: 411-416.

16. Paulino AC, Mai WY, Chintagumpala M, Taher A, The BS (2008) Radiationinduced malignant gliomas: is there a role for reirradiation? Int J Radiat Oncol Biol Phys 71: 1381-1387.

17. Shaw E, Scott C, Souhami L, Dinapoli R, Kline R, et al. (2000) Single dose radiosurgical treatment of recurrent previously irradiated primary brain tumors and brain metastases: final report of RTOG protocol 90-05. Int J Radiat Oncol Biol Phys 47: 291-298.

18. Veninga T, Langendijk HA, Slotman BJ, Rutten EH, van der Kogel AJ, et al. (2001) Reirradiation of primary brain tumours: survival, clinical response and prognostic factors. Radiother Oncol 59: 127-137.

19. Sadikov E, Bezjak A, Yi QL, Wells W, Dawson L, et al. (2007) Value of whole brain re-irradiation for brain metastases--single centre experience. Clin Oncol (R Coll Radiol) 19: 532-538.

20. Emami B, Lyman J, Brown A, Coia L, Goitein M, et al. (1991) Tolerance of norma tissue to therapeutic irradiation. Int J Radiat Oncol Biol Phys 21: 109-122.

21. Lawrence YR, Li XA, el Naqa I, Hahn CA, Marks LB, et al. (2010) Radiation dose-volume effects in the brain. Int J Radiat Oncol Biol Phys 76: S20-27. 